\title{
Comparison of Segmentation-Based Attenuation Correction Methods for PET/MRI: Evaluation of Bone and Liver Standardized Uptake Value with Oncologic PET/CT Data
}

\author{
Joong Hyun Kim ${ }^{1-3}$, Jae Sung Lee ${ }^{1-5}$, In-Chan Song ${ }^{3,6}$, and Dong Soo Lee ${ }^{1-3,7}$ \\ ${ }^{I}$ Department of Nuclear Medicine, Seoul National University, Seoul, Korea; ${ }^{2}$ Interdisciplinary Program in Radiation Applied Life \\ Science, Seoul National University, Seoul, Korea; ${ }^{3}$ Institute of Radiation Medicine, Medical Research Center, Seoul National \\ University, Seoul, Korea; ${ }^{4}$ WCU Department of Brain and Cognitive Sciences, Seoul National University, Seoul, Korea; \\ ${ }^{5}$ Department of Biomedical Sciences, Seoul National University, Seoul, Korea; ${ }^{6}$ Department of Radiology, Seoul National University, \\ Seoul, Korea; and ${ }^{7}$ WCU Department of Molecular Medicine and Biopharmaceutical Science, Seoul National University, \\ Seoul, Korea
}

For attenuation correction (AC) in PET/MRI systems, segmentation-based methods are most often used. However, the standardized uptake value (SUV) of lesions in the bone and liver, which have higher attenuation coefficients than other organs, can be underestimated, potentially leading to misinterpretation of clinical cases. Errors in SUV estimation are also dependent on the segmentation schemes used in the segmentation-based AC. In this study, this potential bias in SUV estimation using 4 different segmentation-based AC methods was evaluated for the PET/CT data of cancer patients with bone and liver lesions. Methods: Forty patients who had spine or liver lesions and underwent ${ }^{18}$ F-FDG PET/CT participated (18 women and 22 men; 20 spine lesions and 20 liver lesions; mean age $( \pm S D), 60.5 \pm$ $11.4 \mathrm{y}$; mean body weight, $57.7 \pm 10.4 \mathrm{~kg}$ ). The patient body region was extracted from the CT image and categorized into 5 tissue groups (air, lungs, fat, water, and bone) using Hounsfield unit thresholds, which were determined from the CT histogram. Four segmentation-based AC methods (SLA [soft-tissue/lung/ air], WFLA [water/fat/lung/air], SLAB [soft-tissue/lung/air/bone], and WFLAB [water/fat/lung/air/bone]) were compared with CTbased AC. The mean attenuation coefficient for each group was calculated from $40 \mathrm{CT}$ images and assigned to the attenuation maps. PET sinograms were reconstructed using segmentationand CT-based AC maps, and mean SUV in the lesions was compared. Results: Mean attenuation coefficients for air, lungs, fat, water, and bone were 0.0058, 0.0349, 0.0895, 0.0987, and $0.1178 \mathrm{~cm}^{-1}$, respectively. In the spine lesions, the SUVs were underestimated by $16.4 \% \pm 8.5 \%$ (SLA AC) and $14.7 \% \pm 7.5 \%$ (WFLA AC) but not to a statistically significant extent for SLAB and WFLAB AC relative to CT AC. In the liver lesions, the SUVs were underestimated by $11.1 \% \pm 2.6 \%, 8.1 \% \pm 3.0 \%, 6.8 \% \pm$ $3.8 \%$, and $4.1 \% \pm 3.8 \%$ with SLA, SLAB, WFLA, and WFLAB $A C$, respectively. Conclusion: Without bone segmentation, the SUVs of spine lesions were considerably underestimated; however, the bias was acceptable with bone segmentation. In liver

Received Feb. 6, 2012; revision accepted Jul. 24, 2012

For correspondence or reprints contact: Jae Sung Lee, Department of Nuclear Medicine, Seoul National University College of Medicine, 101 Daehak-Ro, Jongno-Gu, Seoul 110-744, Korea.

E-mail: jaes@snu.ac.kr

Published online Oct. 18, 2012.

COPYRIGHT (C) 2012 by the Society of Nuclear Medicine and Molecular Imaging, Inc. lesions, the segmentation-based $A C$ methods yielded a negative bias in SUV; however, inclusion of the bone and fat segments reduced the SUV bias. The results of this study will be useful for understanding organ-dependent bias in SUV between PET/CT and PET/MRI.

Key Words: PET/MRI; attenuation correction; standard uptake value (SUV); positron emission tomography

J Nucl Med 2012; 53:1878-1882

DOI: 10.2967/jnumed.112.104109

$\mathbf{H}$ ybrid imaging devices such as PET/CT and SPECT/CT are now widely used in clinical and preclinical studies. Morphologic information provided by CT is particularly useful for the localization of abnormal uptake of radiotracers and for $\gamma$-ray attenuation correction (AC) (1-3). Inaccurate AC of PET and SPECT images can lead to incorrect quantification and misinterpretation of lesions (4-9).

MRI is another important morphologic imaging tool that can be combined with PET or SPECT. MRI has several advantages over CT, including better soft-tissue contrast, no additional radiation hazard, and true 4-dimensional simultaneous imaging (9-11). It is likely that combined PET/MRI and SPECT/MRI systems will facilitate the wider use of radiotracer technologies for clinical and investigational purposes. Several research groups have been successful in demonstrating the feasibility of simultaneous PET/MRI technologies (12-15), and commercial PET/MRI systems with a gantry size sufficient for human whole-body imaging studies have recently become available $(16,17)$.

The intensity of CT images is determined mainly by electron density, which is directly related to the photon linear attenuation coefficient. Thus, the Hounsfield units of CT can be converted into the attenuation coefficient of $511-\mathrm{keV}$ $\gamma$-rays by means of a simple bilinear transformation (1-3). In contrast, MR intensity is associated with proton density or relaxation properties and is therefore not proportional to the 
photon attenuation power. For this reason, it is difficult to derive the $\gamma$-ray attenuation power directly from MR intensity information (18).

For PET AC using MR images, several approaches have been suggested; these methods can be categorized into template-guided AC $(19,20)$ and segmentation-based AC (68 ). For the routine use of template-guided $\mathrm{AC}$, an accurate and robust spatial normalization algorithm is required. For human whole-body PET data, a segmentation-based AC method is regarded as more reliable than template-guided $\mathrm{AC}$ because sometimes the latter cannot accommodate the interpatient variability of anatomic structures in the human torso $(6,7,9,18,21)$.

For segmentation-based AC methods, we segment and categorize tissue groups on the basis of MRI intensity. Usually, 3 (soft-tissue, lungs, and air; no distinction between water-equivalent and fat tissues (6)) or 4 (water-equivalent, fat, lungs, and air; water and fat distinction using Dixon MRI sequence (7)) segmentation-based AC methods are used for torso images. However, bone segmentation has not been as successful in MRI-based torso PET AC until now because it is hard to distinguish bone and air intensities in MR images acquired using standard pulse sequences (9). The relaxation time of cortical bone structures is too short for signal measurements and is a major cause for the similar image intensities of cortical bone and air. Consequently, bone structures were included within the soft-tissue regions in the previously discussed 3 or 4 segmentation-based AC methods.

Recently, several groups have investigated the segmentation of bone structures from soft tissue using the ultra-short time echo (UTE) sequence (22-24). Using the UTE sequence, we can measure early relaxation signals from cortical bone structures. However, more than a $20 \%$ error was introduced in the reconstructed PET images by AC using MR images acquired with the UTE sequence, and some boundary regions between soft tissue and air in the segmented MR images were misinterpreted as bone structures $(23,24)$. The UTE sequence was useful to segment bone only for the head, a relatively simple structure compared with the torso (22).

Using segmentation-based AC methods without bone segmentation, we can expect quantitative inaccuracies in attenuation-corrected PET images (25). In organs with relatively high photon attenuation, uptake of radiotracers would be underestimated. In our preliminary evaluation of organdependent bias in PET tracer uptake, we found that uptake in liver and bone was underestimated in segmentation-based $\mathrm{AC}$ regardless of inclusion of bone segmentation (26). Therefore, in this study, we evaluated the biases in standardized uptake value (SUV) of ${ }^{18}$ F-FDG PET with segmentationbased $\mathrm{AC}$ in patients who had tumor lesions in bone or liver. The accuracies of segmentation-based AC methods used in current commercial PET/MRI systems (SLA [soft-tissue/lung/ air] and WFLA [water/fat/lung/air]) were compared with CTbased AC. Moreover, 2 potential MRI-based AC methods (SLAB [soft-tissue/lung/air/bone] and WFLAB [water/fat/ lung/air/bone]) were included in the comparison under the assumption that the bone structures could be segmented from the soft-tissue group. Abbreviations, number of segments, and related MRI sequences are summarized in Table 1.

\section{MATERIALS AND METHODS}

\section{Subjects and Data Acquisition}

The whole-body PET/CT scan data of 20 liver ( 8 women and 12 men) and 20 spine (10 women and 10 men) oncology patients were retrospectively analyzed. Mean $( \pm \mathrm{SD})$ patient age and weight were $60.5 \pm 11.4$ y (range, $28-81$ y) and $57.7 \pm 10.4 \mathrm{~kg}$ (range, $37-80 \mathrm{~kg}$ ), respectively. The retrospective use of the scan data was approved by the Institutional Review Board of Seoul National University Hospital, Seoul, Korea.

A Biograph TruePoint TrueV PET/CT scanner (Siemens Medical) was used in this study to acquire PET and CT datasets. The scanner comprised 4 rings of detector blocks. Each ring contained 48 detector blocks, and each detector block consisted of a $13 \times 13$ array of lutetium oxyorthosilicate scintillation crystals with an individual dimension of $4 \times 4 \times 20 \mathrm{~mm}$. The axial field of view of the PET scanner was $216 \mathrm{~mm}$. The scanner was operated in 3-dimensional mode for PET emission scans. In all patients, the upper body from neck to upper thigh was covered by a 5- or 6-bed-position emission scan. The duration of the emission scan was $2 \mathrm{~min} / \mathrm{bed}$ position after $60 \mathrm{~min}$ after injection of ${ }^{18} \mathrm{~F}-\mathrm{FDG}(301 \pm 55 \mathrm{MBq}, 5.18 \mathrm{MBq} / \mathrm{kg})$.

\section{Segmented AC Map}

To investigate the SUV bias due to segmentation-based AC, the segmentation-based attenuation maps were generated from the CT images. First, the portion of the CT image representing the patient body was extracted. The Hounsfield unit threshold (-400) was applied to the CT image to extract the patient body and bed automatically, and the patient bed was manually separated from the patient body. Then, the Hounsfield unit histograms were obtained from each patient's CT images, and thresholds between each segment (air, lungs, fat, water, and bone) were determined at the local minima of the histogram. Using the thresholds, patient body parts were automatically classified into 3, 4, or 5 segments (SLA, WFLA, SLAB, and WFLAB). The mean Hounsfield units for each segment were then calculated from $40 \mathrm{CT}$ images and assigned to the body segment of each patient. Finally, the patient bed was added. These images are compared in Supplemental Figure 1 (supplemental materials are available online at http://jnm.snmjournals.org).

\section{Image Reconstruction}

All emission data were reconstructed using these 5 different attenuation maps (CT, SLA, WFLA, SLAB, and WFLAB) and 2-dimensional ordered-subsets expectation maximization with 2 iterations and 14 subsets after random, scatter, attenuation, and normalization corrections and data rebinning. PET reconstruction software (e7 tools) from Siemens Healthcare was used for this purpose. Reconstructed images had dimensions of $168 \times 168 \times 405$ for the 5-bedposition scan and $168 \times 168 \times 479$ for the 6-bed-position scan, with $4.073-\mathrm{mm}$ transaxial pixel spacing and a $2.027-\mathrm{mm}$ axial slice interval.

\section{SUV Comparison}

A region of interest was drawn on metastatic or primary tumor lesions for each PET dataset. For each patient, a single region of interest was applied on a tumor lesion containing the highest-SUV voxel and covering the entire lesion volume. The same region of interest was used for all attenuation-corrected PET images, and mean SUVs were obtained. The SUVs from the segmentation-based 
TABLE 1

Abbreviations of Segmentation-Based AC Methods for PET/MRI That Were Compared in This Study

\begin{tabular}{lcl}
\hline Abbreviation & Number of segments & Segments \\
\hline SLA & 3 & Soft tissue, lung, air \\
WFLA & 4 & Water, fat, lung, air \\
SLAB & 4 & Soft tissue, lung, air, bone \\
WFLAB & 5 & Water, fat, lung, air, bone \\
\hline
\end{tabular}

AC methods (SLA, SLAB, WFLA, and WFLAB AC) were compared with $\mathrm{CT}$ AC by evaluating their percentage differences.

\section{Statistical Analysis}

Paired $t$ tests were performed on SUVs between CT AC and each segmentation-based AC method. Bonferroni adjustment was applied to compensate for multiple comparisons. A $P$ value of 0.05 was used to determine statistical significance.

\section{RESULTS}

For all tissue groups, the mean attenuation coefficients for $511-\mathrm{keV} \gamma$-rays were calculated from the data of 40 patients. The mean attenuation coefficients of air, lung, soft-tissue (fat and water), and bone segments were $0.0058,0.0349,0.0952$ (0.0895 and 0.0987), and $0.1178 \mathrm{~cm}^{-1}$, respectively.

Figure 1 shows difference images of SUV between segmentation-based AC and CT AC. Without bone segmentation, the spine region was considerably underestimated (Figs. $1 \mathrm{~A}$ and $1 \mathrm{C}$ ). Another remarkable finding was that the liver was also underestimated, especially in segmentation-based AC without a distinction between water and fat (Figs. 1A and 1B).

Examples of attenuation-corrected PET images are shown in Figures 2 and 3. Figure 2 shows images of a patient with multiple bone metastases for which attenuation was corrected by the 5 different $\mathrm{AC}$ maps. Without bone segmentation, ${ }^{18} \mathrm{~F}-$ FDG uptake in the lesions on the spine was significantly underestimated (Figs. 2B and 2D); however, the differences do not seem to be significant with bone segmentation (Figs. $2 \mathrm{C}$ and $2 \mathrm{E}$ ). Figure 3 shows images of a patient who had a hypermetabolic lesion in the right lobe of the liver for which attenuation was corrected by the 5 different AC maps. Segmentation-based AC underestimated ${ }^{18} \mathrm{~F}-\mathrm{FDG}$ uptake in comparison with CT-based AC. The error in SUV was greater in segmentation-based AC methods without a water-fat distinction (Figs. 3B and 3C).

For all patient data, the percentage differences in SUV (SLA vs. CT, SLAB vs. CT, WFLA vs. CT, and WFLAB vs. $\mathrm{CT}$ ) were quantified (Fig. 4). In spine (bone) lesions, the SUVs were underestimated by $16.4 \% \pm 8.5 \%$ (SLA, $P<$ $10^{-6}$ ) and $14.7 \% \pm 7.5 \%$ (WFLA, $P<10^{-7}$ ) with segmentation-based AC without bone segmentation, but the difference was not statistically significant with segmentation-based AC with bone segmentation $(P>0.05$ for both SLAB and WFLAB). In liver lesions, the SUVs were underestimated by $11.1 \% \pm 2.6 \%\left(P<10^{-9}\right), 8.1 \% \pm 3.0 \%\left(P<10^{-8}\right)$, $6.8 \% \pm 3.8 \%\left(P<10^{-6}\right)$, and $4.1 \% \pm 3.8 \%\left(P<10^{-3}\right)$ with SLA, SLAB, WFLA, and WFLAB AC, respectively.

\section{DISCUSSION}

$\mathrm{AC}$ is a necessary process for the reconstruction of PET images. In particular, accurate AC is essential for quantitative studies such as of tracer kinetics or radiation dosimetry and for clinical follow-up studies in which the severity of cancer is evaluated using the mean or maximum SUV. However, in PET/MRI systems, the AC map cannot be obtained directly from MRI because voxel intensities in MR images are not related to photon attenuation coefficients and are not quantitative (i.e., they are expressed in arbitrary units) (21). For PET/MRI combined systems, therefore, attenuation coefficients

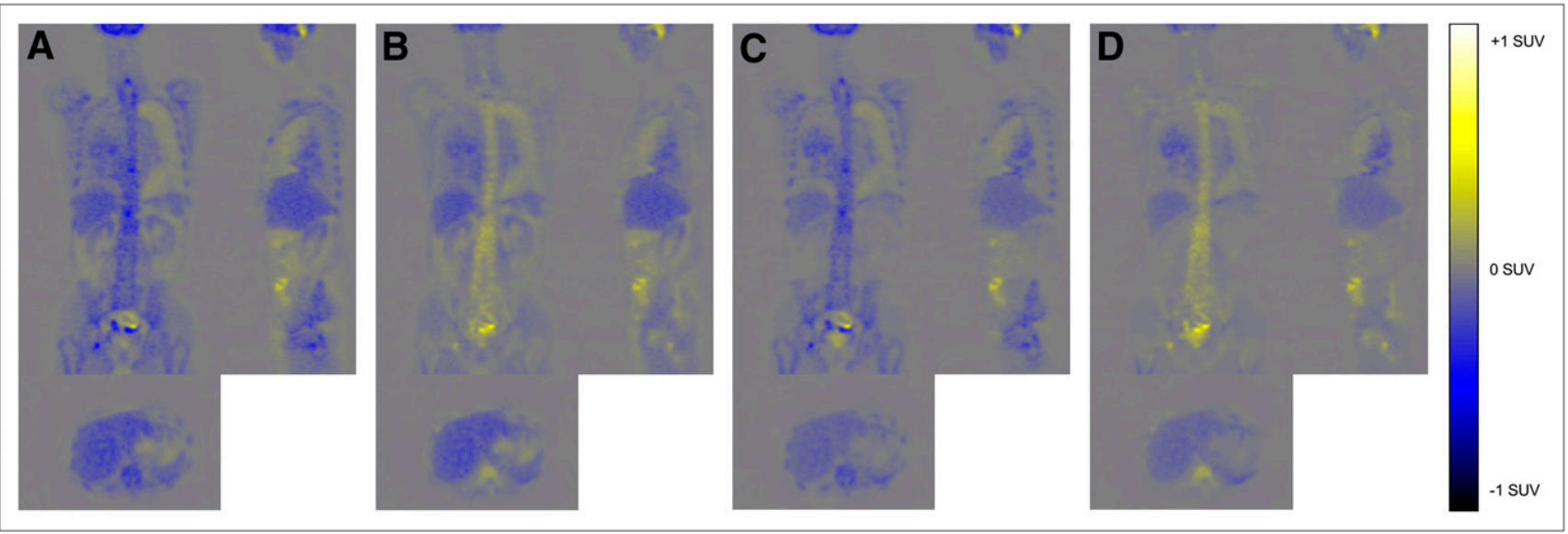

FIGURE 1. SUV difference images between SLA and CT AC (A), between SLAB and CT AC (B), between WFLA and CT AC (C), and between WFLAB and CT AC (D). 

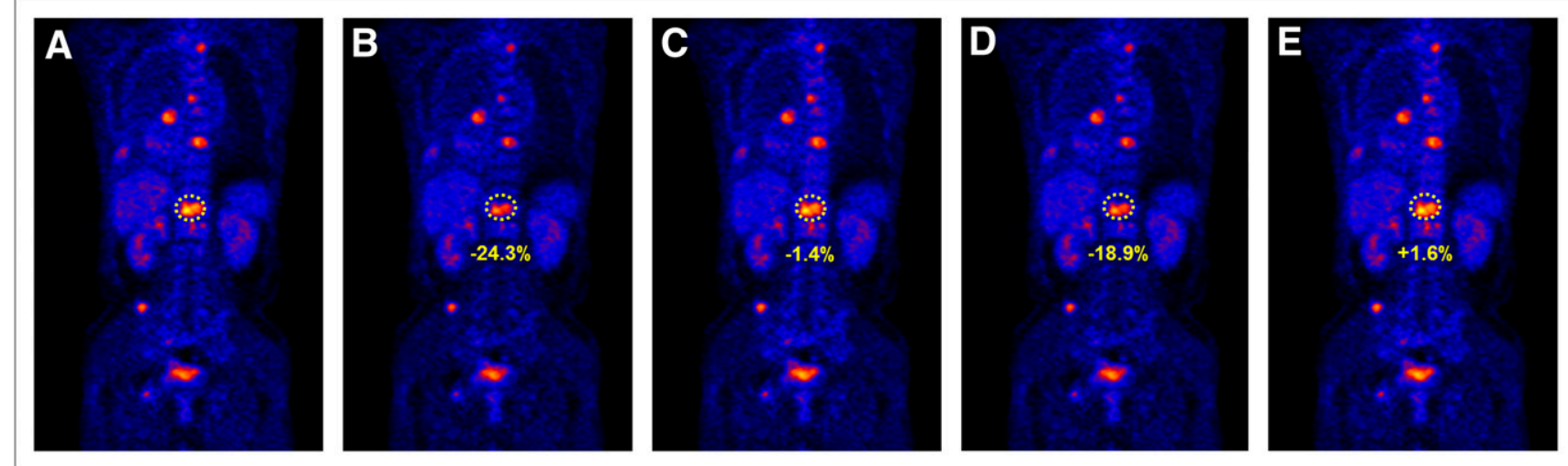

FIGURE 2. Attenuation-corrected PET data of patient with multiple bone lesions using CT (A), SLA (B), SLAB (C), WFLA (D), and WFLAB (E). Values below marked lesions are percentage differences in SUV relative to CT AC.

have to be estimated and not measured. The most reliable candidate method for MRI-based AC is segmentation-based AC. This method assumes homogeneous attenuation power in a segment. However, the segmentation-based AC method distinguishes only a few regions, and as a result, each segment contains multiple organs of differing photon attenuation. Organ-dependent bias is the result of this assumption.

For segmentation-based $\mathrm{AC}$ without bone segmentation, we observed considerable negative bias $(-16.4 \%$ and $-14.7 \%$ for SLA AC and WFLA AC, respectively) in spine cancer lesions mainly because the bone structures, which have high-attenuating components, were regarded as soft tissues, which have low-attenuating components. In a previous investigation on the effects of bone segmentation using beagles (8), 3-segment-based AC yielded approximately $10 \%$ bias in the spine region, and this bias decreased to $3 \%$ if bone segmentation was applied. In addition, there was a recent report that substitution of bone by soft tissue in the data of 22 patients introduced tracer uptake underestimations in osseous regions by $11.2 \%$ (27). In this study, we confirmed this bone segmentation effect in human data. Therefore, bone segmentation is a fundamental process for accurate quantification of bone regions.

The UTE sequence provides the possibility of accurate bone segmentation. Until recently, however, the UTE sequence was not a perfect solution for bone segmentation, especially in the torso. Images obtained with UTE have high noise because of the short relaxation time of cortical bone structures. To improve image quality, the acquisition time should be lengthened because repetitive acquisitions are required. Another main limitation for UTE in body imaging is the difficulty in achieving a homogeneous magnetic field across a large field of view. Moreover, streak artifacts and misinterpretation of body edges as bone are further challenges in using UTE sequences for AC of PET images $(22,24)$. All these limitations are more severe for thorax and pelvis imaging than for head and neck imaging. Consequently, most UTE studies are focused on brain studies (22-24).

In this study, we also evaluated SUV in the liver. The results shown in Figures 3 and 4 indicate that both bone segmentation and water-fat distinction contribute to the reduction of SUV bias in the liver. Although the combination of these 2 techniques (WFLAB AC) reduced bias to less than $5 \%$, the negative bias was statistically significant. This bias might be caused by the same processes as in bone lesions corrected with segmentation-based AC without bone segmentation. The density of the liver is relatively higher than that of other tissue organs. The mean attenuation coefficient of the liver calculated from a region of interest on the liver in our 40 subjects was higher $\left(0.1018 \mathrm{~cm}^{-1}\right)$

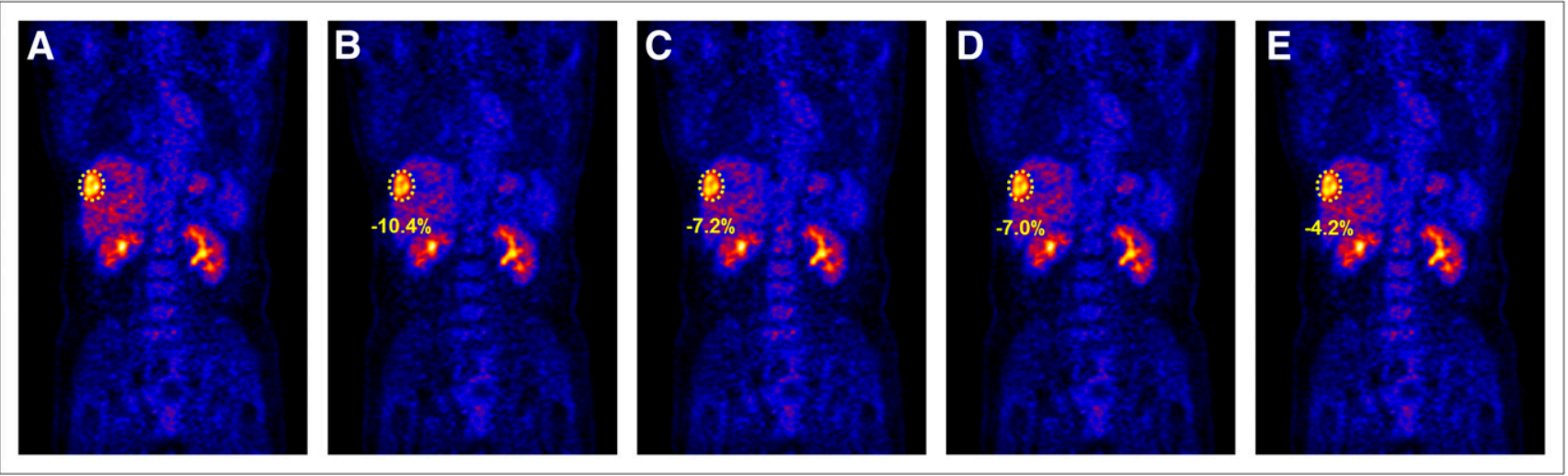

FIGURE 3. Attenuation-corrected PET data of patient with hepatic lesion using CT (A), SLA (B), SLAB (C), WFLA (D), and WFLAB (E). Values below marked lesions are percentage differences in SUV relative to CT AC. 


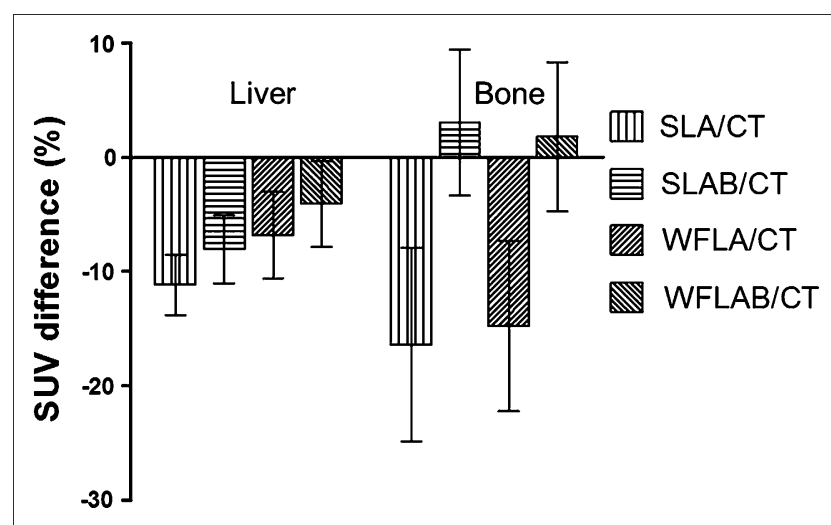

FIGURE 4. SUV percentage differences in segmentation-based AC compared with CT AC for bone and liver lesions.

than that of the water segment. Thus, organ-dependent bias according to organ density will exist if various soft tissues are regarded as a single tissue group. Organ-dependent bias would be an unavoidable limitation in quantification studies using segmentation-based AC in PET/MRI.

The assignment of a nonzero attenuation coefficient $(0.0058$ $\mathrm{cm}^{-1}$ ) to air in this study would not reflect reality and reduce the bias with respect to the reference $\mathrm{CT}$ reconstruction.

\section{CONCLUSION}

Segmentation-based AC without bone segmentation caused a considerable underestimation of the SUV of spine lesions; however, with bone segmentation, the bias was acceptable. In liver lesions, segmentation-based AC methods yielded negative bias in SUV; however, inclusion of the bone and fat segments reduced the SUV bias. The results of this study will be useful for understanding the organ-dependent bias in SUV between PET/CT and PET/MRI.

\section{DISCLOSURE STATEMENT}

The costs of publication of this article were defrayed in part by the payment of page charges. Therefore, and solely to indicate this fact, this article is hereby marked "advertisement" in accordance with 18 USC section 1734.

\section{ACKNOWLEDGMENT}

This work was supported by grants 800-20110287, 20082003852, 2010-0026012, and R31-10089 (WCU program) from the Ministry of Knowledge Economy and KOSEF, funded by the Ministry of Education, Science, and Technology. No other potential conflict of interest relevant to this article was reported.

\section{REFERENCES}

1. Burger C, Goerres G, Schoenes S, Buck A, Lonn AH, Von Schulthess GK. PET attenuation coefficients from CT images: experimental evaluation of the transformation of CT into PET 511-keV attenuation coefficients. Eur J Nucl Med Mol Imaging. 2002;29:922-927.

2. Kinahan PE, Hasegawa BH, Beyer T. X-ray-based attenuation correction for positron emission tomography/computed tomography scanners. Semin Nucl Med. 2003;33:166-179.
3. Carney JP, Townsend DW, Rappoport V, Bendriem B. Method for transforming CT images for attenuation correction in PET/CT imaging. Med Phys. 2006;33:976983.

4. Boss A, Bisdas S, Kolb A, et al. Hybrid PET/MRI of intracranial masses: initial experiences and comparison to PET/CT. J Nucl Med. 2010;51:1198-1205.

5. Hofmann M, Bezrukov I, Mantlik F, et al. MRI-based attenuation correction for whole-body PET/MRI: quantitative evaluation of segmentation- and atlas-based methods. J Nucl Med. 2011;52:1392-1399.

6. Schulz V, Torres-Espallardo I, Renisch S, et al. Automatic, three-segment, MRbased attenuation correction for whole-body PET/MR data. Eur J Nucl Med Mol Imaging. 2011;38:138-152.

7. Martinez-Möller A, Souvatzoglou M, Delso G, et al. Tissue classification as a potential approach for attenuation correction in whole-body PET/MRI: evaluation with PET/CT data. J Nucl Med. 2009;50:520-526.

8. Steinberg J, Jia G, Sammet S, Zhang J, Hall N, Knopp MV. Three-region MRIbased whole-body attenuation correction for automated PET reconstruction. Nucl Med Biol. 2010;37:227-235.

9. Hofmann M, Pichler B, Schölkopf B, Beyer T. Towards quantitative PET/MRI: a review of MR-based attenuation correction techniques. Eur J Nucl Med Mol Imaging. 2009;36(suppl 1):S93-S104.

10. Lee JS, Kang KW. PET/MRI. In: Kim EE, Lee MC, Inoue T, Wong W-H, eds. Clinical PET and PET/CT: Principles and Applications. 2nd ed. Berlin, Germany: Springer; 2011.

11. Wehrl HF, Judenhofer MS, Wiehr S, Pichler BJ. Pre-clinical PET/MR: technological advances and new perspectives in biomedical research. Eur J Nucl Med Mol Imaging. 2009;36(suppl 1):S56-S68.

12. Judenhofer MS, Wehrl HF, Newport DF, et al. Simultaneous PET-MRI: a new approach for functional and morphological imaging. Nat Med. 2008;14:459-465.

13. Catana C, Procissi D, Wu Y, et al. Simultaneous in vivo positron emission tomography and magnetic resonance imaging. Proc Natl Acad Sci U S A. 2008;105:37053710 .

14. Yamamoto S, Imaizumi M, Kanai $\mathrm{Y}$, et al. Design and performance from an integrated PET/MRI system for small animals. Ann Nucl Med. 2010;24:89-98.

15. Yoon HS, Ko GB, Kwon SI, et al. Initial results of simultaneous PET/MRI experiments with an MRI-compatible silicon photomultiplier PET scanner. J Nucl Med. 2012;53:608-614.

16. Zaidi H, Ojha N, Morich M, et al. Design and performance evaluation of a whole-body Ingenuity TF PET-MRI system. Phys Med Biol. 2011;56:30913106.

17. Delso G, Fürst S, Jakoby B, et al. Performance measurements of the Siemens mMR integrated whole-body PET/MR scanner. J Nucl Med. 2011;52:19141922.

18. Hofmann M, Steinke F, Scheel V, et al. MRI-based attenuation correction for PET/MRI: a novel approach combining pattern recognition and atlas registration. J Nucl Med. 2008;49:1875-1883.

19. Malone IB, Ansorge RE, Williams GB, Nestor PJ, Carpenter TA, Fryer TD. Attenuation correction methods suitable for brain imaging with a PET/MRI scanner: a comparison of tissue atlas and template attenuation map approaches. J Nucl Med. 2011;52:1142-1149.

20. Schreibmann E, Nye JA, Schuster DM, Martin DR, Votaw J, Fox T. MR-based attenuation correction for hybrid PET-MR brain imaging systems using deformable image registration. Med Phys. 2010;37:2101-2109.

21. Beyer T, Weigert M, Quick HH, et al. MR-based attenuation correction for torsoPET/MR imaging: pitfalls in mapping MR to CT data. Eur J Nucl Med Mol Imaging. 2008;35:1142-1146.

22. Johansson A, Karlsson M, Nyholm T. CT substitute derived from MRI sequences with ultrashort echo time. Med Phys. 2011;38:2708-2714.

23. Catana C, van der Kouwe A, Benner T, et al. Toward implementing an MRIbased PET attenuation-correction method for neurologic studies on the MR-PET brain prototype. J Nucl Med. 2010;51:1431-1438.

24. Keereman V, Fierens Y, Broux T, De Deene Y, Lonneux M, Vandenberghe S. MRI-based attenuation correction for PET/MRI using ultrashort echo time sequences. J Nucl Med. 2010;51:812-818.

25. Schleyer PJ, Schaeffter T, Marsden PK. The effect of inaccurate bone attenuation coefficient and segmentation on reconstructed PET images. Nucl Med Commun. 2010;31:708-716.

26. Kim JH, Lee JS, Song I-C, Lee DS. Accuracy evaluation of four-segment wholebody attenuation correction in image based radiation dosimetry using PET/MRI. Presented at: IEEE Nuclear Science Symposium Medical Imaging Conference; November 5, 2010; Knoxville, Tennessee.

27. Samarin A, Burger C, Wollenweber SD, et al. PET/MR imaging of bone lesions: implications for PET quantification from imperfect attenuation correction. Eur J Nucl Med Mol Imaging. 2012;39:1154-1160. 